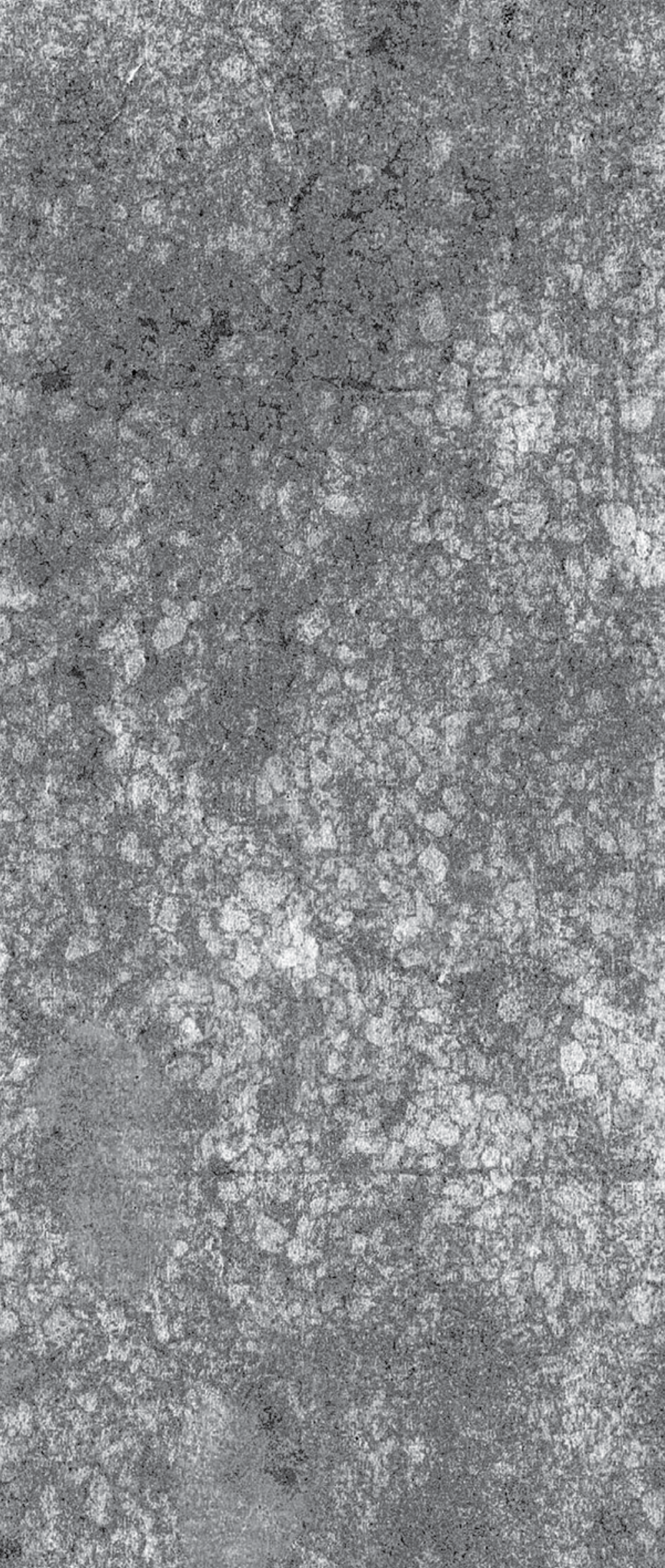

1. Arquiteto urbanista, doutor em Geografia Tratamento da Informação Espacial no Programa de Pós-Graduação em Geografia - Tratamento da Informação Espacial da PUC Minas, coordenador do Escritório de Integração - DAU/PUC Minas, professor do Departamento de Arquitetura e Urbanismo da PUC Minas

2. Arquiteto urbanista, especialista em Planejamento Ambiental Urbano (PUC Minas). Também participam do projeto os alunos da PUC Minas: Alecsandra Cunha (Geografia), Bruna Oliveira (Administrativo), Lisandra Silva (Arquitetura e Urbanismo), Luana Maíra (Direito) e Renata Duarte (Arquitetura e Urbanismo).

3. Professor dos cursos de Engenharia Ambiental e Sanitária e Engenharia Civil da Faculdade Pitágoras - Betim, Diretor Geral do Instituto de Planejamento Urbano da Prefeitura de Contagem/MG. Mestrando do Curso de Pós-Graduação em Ciências Sociais da PUC Minas e Pesquisador do Núcleo Jurídico de Políticas Urbanas - NUJUP/ PUC Minas.

4. Engenheira Arquiteta, doutora pelo Programa de Pós-Graduação em Arquitetura e Urbanismo pela Universidade Federal de Minas Gerais, Mestre em Engenharia de Construções Civis e Urbanas pela Universidade de São Paulo, professora do curso de Arquitetura e Urbanismo e integrante da equipe do Escritório de Integração da PUC Minas; professora da Escola de Arquitetura da UFMG 


\title{
DESEQUILÍBRIO AMBIENTAL, CULTURA LOCAL E CULTURA \\ TECNOLÓGICA: A PARCERIA \\ ENTRE A ASSOCIAÇÃO \\ DOS MORADORES DO \\ JARDINS DE PETRÓPOLIS EO \\ ESCRITÓRIO DE INTEGRAÇÃO DO DAU DA PUC MINAS
}

\section{ENVIRONMENTAL IMBALANCE, LOCAL CULTURE AND TECHNOLOGICAL CULTURE: THE PARTNERSHIP BETWEEN THE ASSOCIATION OF JARDINS DE PETRÓPOLIS RESIDENTS AND THE ESCRITÓRIO DE INTEGRAÇÃO DO DAU DA PUC MINAS}

Alfio Conti ${ }^{1}$

Danilo de Carvalho Botelho Almeida² Marco Antonio Souza Borges Netto ${ }^{3}$ Margarete Maria de Araújo Silva ${ }^{4}$

\section{Resumo}

O objetivo desse texto é apresentar uma experiência de requalificação ambiental urbana em curso no município de Nova Lima. O objeto de estudo é uma área lindeira ao condomínio Jardins de Petrópolis, que é afetada por graves processos de degradação físico-ambiental. A partir de uma parceria entre a Associação de moradores locais e o Escritório de Integração da PUC Minas, a proposta enfoca o resgate da cultura tecnológica local, para que dispositivos específicos sejam aplicados dentro de uma abordagem sistêmica, usando mão de obra local em toda a área de intervenção.

Palavras-chave: Degradação ambiental, requalificação urbana, extensão universitária

\begin{abstract}
The aim of this paper is to present an experience of urban environmental restoration underway in Nova Lima. The object of study is an area of the neighborhood Jardins de Petrópolis, which is affected by severe physical degradation processes and environmental. From a partnership between the local association residents and the Escritório de Integração da PUC Minas, the proposal focuses on the rescue of the local technological culture, so the specific devices designed could be applied in a systematic approach, using local labor throughout the intervention area.
\end{abstract}

Key words: Environmental degradation, urban renewal, extension education 
O objetivo deste texto é apresentar uma experiência de requalificação ambiental urbana em curso no Município de Nova Lima. O objeto de estudo é uma área lindeira ao condomínio Jardins de Petrópolis, localizado na parte central do Município, a sudoeste da mancha urbana correspondente ao núcleo urbano sede do Município, que é afetada por graves processos de degradação físico-ambiental. Na área, são encontradas, de maneira difusa e generalizada, erosões com ravinamento e voçorocamento, atingindo o lençol freático e as redes de mesoestruturas implantadas, com o assoreamento dos cursos d'água e a destruição da cobertura vegetal.

O processo de requalificação ambiental urbana é objeto de um projeto de extensão financiado pela Pró-Reitoria de Extensão da Pontifícia Universidade Católica de Minas Gerais (Proex/PUC Minas), que tem, entre outros, o objetivo de implantar uma cooperação tecnológico-cultural entre a Associação dos Moradores do Bairro Jardins de Petrópolis e o Escritório de Integração do Departamento de Arquitetura e Urbanismo da Pontifícia Universidade Católica de Minas Gerais (EI/DAU/PUC Minas). A proposta de recuperação do equilíbrio ambiental da área da Cascalheira enfoca o resgate da cultura tecnológica local, para que dispositivos específicos sejam aplicados dentro de uma abordagem sistêmica, usando mão de obra local em toda a área de intervenção.

A partir dos problemas identificados, a metodologia prevê a elaboração de um estudo integrado sobre as origens e as manifestações dos problemas existentes, visando à busca de soluções ambientalmente compatíveis e de baixo impacto, junto à comunidade local. As soluções, assim como as medidas previstas para o desenvolvimento e implantação do processo de recuperação e reequilíbrio ambiental serão realizadas pela Associação, em parceria com a Prefeitura Municipal de Nova Lima.

As propostas, o projeto e a evolução do processo de recuperação serão sistematizados e divulgados por meio da criação de materiais didáticos para que, além de serem adotados na área, possam servir de referência para lugares onde se manifestam as mesmas patologias, que são recorrentes também nos assentamentos informais.

\section{Considerações críticas e análise teórica}

No processo de urbanização brasileiro, a distribuição espacial da população reflete a distribuição das alternativas de acesso à cidade no que concerne à moradia e aos produtos imobiliários a ela relacionados, disponíveis à população, segundo a faixa de renda. Uma parte da sociedade, nesse caso, os grupos de renda média e alta, buscou soluções específicas. Na Região Metropolitana de Belo Horizonte, segundo Moura, esses grupos foram ocupando "um crescente número de loteamentos fechados, misto de residência e de casa de campo, aprazíveis espaços, característicos de um tipo privilegiado de crescimento da cidade para além da Serra do Curral, rumo ao sul" (MOURA, 1994, p. 71).

O condomínio Jardins de Petrópolis é um desses produtos e vem sofrendo os impactos ambientais gerados pela adoção de soluções urbanísticas (frequentemente empregadas na cidade 
formal) que ignoram as especificidades locais e os condicionantes ambientais. Essas soluções não desempenham corretamente seu papel, gerando impactos negativos, cuja entidade varia de acordo com o tempo e com uma progressão que é, em geral, cumulativa, potencializando os problemas e os estragos.

O resultado, em geral, é o desequilíbrio hidrogeológico do meio ambiente. É um problema real que afeta o território brasileiro da mesma maneira que outras realidades espalhadas em todo o mundo. As catástrofes e os prejuízos humanos e materiais, tantas vezes objeto de manchetes por parte da mídia, são os efeitos mais evidentes desse problema. Na realidade, desastres naturais são os resultados, muitas vezes, da acumulação e da sedimentação do desequilíbrio em consequência de ações desarticuladas e equivocadas. Esse processo de evolução do desequilíbrio ambiental é o fruto de posturas e atitudes cujas raízes mais profundas encontram-se na própria cultura das populações afetadas.

Por outro lado, a cultura local e as práticas associadas à ocupação, implantação e gestão dos assentamentos humanos são elementos que caracterizam e distinguem cada civilidade. A história nos ensina que essas práticas evoluem lenta e progressivamente e são frutos de ajustes contínuos e pormenorizados. Além do mais, as ações de intervenção devem ser feitas em uma escala que possa ser dominada, para controlar efeitos não previstos. Nesse sentido, cabe lembrar a história de Veneza e da sua laguna, onde, ao longo dos séculos, os venezianos, por questões de sobrevivência, empenharam-se em compreender as características e os processos mais íntimos que regulavam o ecossistema da laguna, ao centro da qual sua cidade estava implantada. Esse conhecimento permitiu-lhes equilibrar esse ecossistema instável por natureza, evitando os cenários finais que seriam, de um lado, o assoreamento (e isso foi evitado, desviando-se para o mar uma boa parte daqueles rios que desembocavam na laguna) e, do outro, a invasão do mar (que foi evitada implantando-se engenhosas obras de engenharia hidráulica). Os notórios problemas que a cidade e seus habitantes vivem atualmente são de origem contemporânea e resultado da perda desse conhecimento ao longo dos últimos 200 anos.

No caso brasileiro, as práticas associadas à ocupação dos assentamentos humanos remontam ao processo de colonização portuguesa. E o esforço inicial de adequação das soluções práticas, dispositivos, características e condicionantes ambientais do Novo Mundo foram se perdendo, sendo substituídos por uma importação exógena e acrítica de ideias, conceitos, práticas e tecnologia. Essa postura, que vem se reproduzindo para todos os campos do conhecimento, instala-se ao longo de toda a história brasileira, tornando-se parte integrante de uma cultura dissociada do conhecimento íntimo da realidade dos processos físico-ambientais. A sustentabilidade ambiental, no seu sentido mais amplo, se traduz no domínio e na construção desse conhecimento; contudo, apesar de essa consciência existir, os acontecimentos do dia a dia mostram o quanto a aplicação desse conceito encontra-se longe de ser uma realidade.

Atualmente não é mais suficiente manifestar-se favorável a certos tipos de ideias; é imprescindível tomar atitudes e implantar 
ações, programas e criar uma cultura pelo fazer pragmático. As soluções existem e estão disponíveis. Nunca como hoje foi tão fácil ter acesso ao conhecimento no seu sentido mais amplo. Entretanto, essa disponibilidade de conhecimentos ainda não se transformou em melhorias qualitativas do nosso habitat.

A percepção de que os problemas ambientais e sociais são interligados tem aumentado, gerando a necessidade do resgate de uma abordagem holística da questão, conforme sugere a Declaração do Rio sobre Meio Ambiente e Desenvolvimento, bem como a Seção IV C-5 da Agenda Habitat, relativa ao Desenvolvimento Sustentável dos Assentamentos Humanos em um Mundo Urbano.

No Brasil, a Lei federal $n^{\circ} 10.257$, de 10 de julho de 2001, que instituiu o Estatuto da Cidade, estabeleceu as diretrizes gerais da política urbana. Algumas delas seguem parâmetros socioambientais, tais como:

- a garantia do direito a cidades sustentáveis, entendido como o direito à terra urbana, à moradia, ao saneamento ambiental, à infraestrutura urbana, ao transporte e aos serviços públicos, ao trabalho e ao lazer, para as presentes e futuras gerações;

- a proteção do meio ambiente natural e constituído do patrimônio cultural, histórico, artístico, paisagístico e arqueológico;

- a regularização fundiária e a urbanização de áreas ocupadas por populações de baixa renda, mediante o estabelecimento de normas especiais de urbanização, uso e ocupação do solo e edificação, consideradas a situação socioeconômica da população e as normas ambientais.

As recomendações dos documentos supramencionados envolvem a elaboração de estudos ambientais e de impacto ambiental para planos e projetos de desenvolvimento que afetem significativamente a qualidade do meio ambiente. Também preveem a incorporação, de modo integrado, de princípios e estratégias contidos na Agenda 21, tais como: o princípio da precaução, o princípio do poluidor pagador, o princípio da prevenção à poluição e a aplicação de uma abordagem ecossistêmica; a promoção da conservação e do uso sustentado da biodiversidade existente no perímetro urbano; a ampla participação de todas as partes interessadas no planejamento espacial, dentre outros.

Para a concretização do processo de requalificação urbana do trecho escolhido na área da Cascalheira, é importante descrever alguns princípios acima citados do Direito Ambiental. Quais sejam:

\section{Princípio da participação comunitária}

Esse princípio, presente no caput do artigo 225 da Constituição Federal de 1988, não se aplica somente ao Direito Ambiental. Fundamenta-se na concepção de que, para que sejam instituídas políticas ambientais, bem como os assuntos discutidos de forma salutar, é fundamental a cooperação entre o Estado e a comunidade. O sucesso nos resultados demonstra que tanto a população quanto a força sindical têm se envolvido ativamente em definir e realinhar tais políticas.

\section{Princípio do poluidor pagador}

Nesse princípio, os agentes econômicos, sejam públicos ou privados, devem calcular o custo social da poluição por eles gerada, 
e este deve ser assumido ou internalizado. Isso acontece porque, junto com o processo produtivo, também são produzidas externalidades negativas. Esse nome se dá pelo fato de que os resíduos da produção são recebidos pela coletividade, enquanto o lucro é recebido somente pelo produtor. Não se deve confundir esse princípio como licença para poluir, pois o ônus para o poluidor tem caráter punitivo, pois o meio ambiente deve ser preservado, inclusive no processo de produção e desenvolvimento.

No caso do condomínio Jardins de Petrópolis, a exploração de cascalho causou dano à área e, por causa disso, foram tomadas providências. Porém o trabalho desenvolvido na época foi ainda mais danoso com o passar do tempo, refletindo-se em outras áreas, conforme veremos.

\section{Princípio da prevenção}

Édis Milaré (2001) define que "o princípio da prevenção é basilar ao Direito Ambiental, concernindo à prioridade de que devem ser dadas as medidas que evitem o nascimento de atentados ao ambiente, molde a reduzir ou eliminar as causas de ações suscetíveis de alterar a sua qualidade". Isso vale dizer que, segundo esse princípio, as possíveis ações danosas ao meio ambiente devem ser identificadas e eliminadas antes de se concretizarem, em proteção à sociedade atual e futura.

Compete à Administração Pública zelar pela boa qualidade de vida da sociedade, isto é, pelo interesse público. Uma vez que o meio ambiente ecologicamente equilibrado, assim como a efetivação dos direitos sociais constituem elementos fundamentais garantidores da boa qualidade de vida, o Poder Público deve atuar nesse sentido. Por isso, entendemos que o papel do Poder Público é fundamental, desde que atue não isoladamente.

Portanto o processo de requalificação ambiental urbana deve recuperar as erosões e impedir que elas se alastrem, causando maiores transtornos ao meio ambiente e aos residentes da área.

\section{Contextualização e considerações crítico-propositivas}

A população-alvo da proposta se compõe pelos moradores do Bairro Jardins de Petrópolis e pelas comunidades localizadas na bacia hidrográfica dos córregos dos Macacos e Flor-de-Lis. É formada em sua maioria por famílias de renda média, cujos chefes de família trabalham, em geral, em Belo Horizonte, como funcionários públicos, empresários ou profissionais liberais. Também há a presença de população de baixa renda que trabalha na agricultura de subsistência ou no setor terciário com baixa qualificação, sendo uma boa parte deles empregados pelas famílias moradoras do Bairro Jardins de Petrópolis. O projeto beneficia diretamente toda a população residente na área, estimada em mais de 2 000 pessoas, e, indiretamente, a população que mora nas bacias dos córregos acima mencionados. À jusante da área de intervenção, a população é estimada em torno de 3000 pessoas.

A área denominada como "Cascalheira", localizada em terreno de propriedade particular, inicialmente sem acesso direto, é conhecida por esse nome devido ao processo de extração mineral ali implantado. Devido ao tipo de atividade, foi necessária a abertura de uma via com recursos do Poder Público, ligando a Avenida Morro 
do Pires ao local, que continuou até recentemente com pouco ou nenhum controle no que diz respeito ao impacto ambiental. A exploração de minério foi efetivada sem prever um processo de recuperação da área, retirando o extrato superficial da canga e deixando exposta a formação geológica subjacente de xisto, que é muito susceptível à erosão por parte dos agentes atmosféricos. Os impactos gerados podem desenvolver desequilíbrios ambientais por uma alteração físico-ambiental deficitária de controle e previsão de seus efeitos no meio ambiente, em razão da não compreensão de seu funcionamento e dos impactos gerados pelas soluções tecnológicas tradicionais ali implantadas. Estes resultam na criação e evolução de processos patológicos, como erosões e assoreamentos, afetando, em primeiro lugar, o sistema das águas, que, por sua vez, potencializa tais processos, afetando os lotes e as edificações do Bairro Jardins de Petrópolis na sua porção sul e sudeste.

No ano de 1997 ou 1998, segundo informações dos moradores locais, foi feito um projeto de recuperação da área degradada do Morro do Pires pela empresa Eneplan - Projeto e Consultorias Ltda. Tal projeto consistiu na implantação de grandes platôs, limitados por canaletas com o escopo de direcionar a água para uma bacia de contenção de finos. Essa bacia encontra-se implantada, aproximadamente, na cota 980 e está confinada por um muro de pneus. A água da bacia de retenção é direcionada por tubulação até uma escada dissipadora. Os dispositivos e as soluções adotadas por essa intervenção de recuperação, que visou a disciplinar as águas, hoje se encontram, em grande parte, destruídos pela ação da própria água, servindo eles próprios como fator para o desencadeamento de novos processos erosivos. Devido às soluções adotadas, esse projeto promoveu a concentração e o aumento da velocidade das águas pluviais, acarretando problemas à jusante, verificados após a escada dissipadora. Contornando o Morro do Pires no sentido de Nova Lima, também se observam erosões transversais potencializando as erosões longitudinais à via em sulcos profundos, e, nos terrenos adjacentes, afetando o Bairro Jardim de Petrópolis. As ruas do loteamento não têm nenhum tipo de pavimentação, apresentando problemas originados à montante da área em questão, como as soluções de drenagem pluvial adotadas, incompatíveis com as características geológicas e geométricas das ruas, assim como com as condicionantes físico-ambientais.

Os processos patológicos mencionados acima extrapolaram a área da "Cascalheira", que ocupa uma porção da vertente sul do Morro do Pires, em uma região de meia encosta, afetando as áreas à jusante da Avenida Morro do Pires (arruamento e loteamento). A área entre a via de acesso à "Cascalheira" e a área de abrangência do projeto da Eneplan acabou por se tornar uma área residual. Sem tratamento algum, esta apresenta focos de erosão e, com isso, alavancam os problemas citados anteriormente.

A visão equivocada, mas ainda muito presente nas propostas de intervenção ambiental, de elaborar um projeto que solucione e reequilibre quase que instantaneamente uma situação que veio se conformando ao longo de anos, não faz parte das intenções desta proposta. Conforme vimos, a área já foi objeto de uma intervenção que adotou uma visão equivocada e ficou materialmente evidente como isso não deu certo, tornando-se, paradoxalmente, um fator a mais de risco.

O conceito básico a ser utilizado no projeto é minimizar ou anular os efeitos negativos (impactos) das ações antropogênicas 
executadas de maneira inconsequente sobre o meio físico e o meio ambiente, buscando devolver o equilíbrio ecossistêmico ao local. Para isso, é necessário priorizar a definição e implantação de um processo gradual de reequilíbrio, pautado numa ação emergencial e num conjunto de ações de médio e longo prazo para o manejo da área, a fim de atingir seu novo equilíbrio.

É importante destacar a necessidade de realizar um processo em etapas, com monitoramento e controle, para atingir os objetivos previstos, pois isso possibilitaria importantes e necessários ajustes ao longo do processo, como parte integrante da própria implantação.

\section{Conclusão}

A ideia norteadora é a implantação de um processo que incorpore dimensão do monitoramento, controle e gestão, diluindo, ao longo dele, os impactos das soluções propostas, por meio dos necessários ajustes vistos como etapas estratégicas para o reequilíbrio ambiental do lugar. Com isso, não se pretende não atacar a situação atual de emergência; pelo contrário, para ela serão previstas medidas de impacto necessárias para estancar as situações mais graves. As medidas consideradas de impacto nessa fase emergencial nada terão a ver com as previstas em propostas consideradas usuais, mas deverão utilizar tecnologias ambientalmente sustentáveis, que possam aproveitar dos recursos renováveis presentes no local da intervenção, buscando, assim, reequilibrar o sistema das águas, desenvolvendo soluções de drenagem pluvial que priorizem a dispersão e a infiltração; minimizando seus impactos, como a ampliação dos fenômenos erosivos existentes e a continuação do processo de assoreamento e a destruição das mesoestruturas implantadas nas áreas a jusante.

Este trabalho vem sendo desenvolvido pelo El/DAU/PUC Minas desde o ano de 2004, em parceria com a Associação dos Moradores do Bairro Jardins de Petrópolis. Sendo assim, este projeto se configura como a continuação de um trabalho pautado no conhecimento dos reais problemas abordados e nas soluções possíveis de recuperação da área em questão, e pretende consolidar e constituir uma importante fonte para sistematizar e circular o conhecimento produzido entre a comunidade acadêmica e a comunidade local.

\section{Referências}

MILARÉ, Edis. Direito do ambiente: doutrina, prática, jurisprudência, glossário. 2. ed. São Paulo: Revista dos Tribunais, 2001.

MOURA, Heloísa Soares de. Habitação e produção do espaço em Belo Horizonte. In: MONTE-MÓR, Roberto de Mello. (Org.) Belo Horizonte: espaços e tempos em construção. Belo Horizonte: Cedeplar, 1994.

\section{Endereço para correspondência}

Alfio Conti

contialfio@gmail.com

Danilo de Carvalho Botelho Almeida

danilo.botelho@gmail.com

Margarete Maria de Araújo Silva

leta123@gmail.com

Marco Antonio Souza Borges Netto

marcoborgesnetto@gmail.com 